minor renal anomalies are removed from Luck's figures the sensitivity falls to just under $70 \%$; this is the sort of figure obtained in many othe departments and, I believe, the one that should be quoted when talking about the overall sensitivity of routine ultrasound.

One other factor that Luck did not make clea was the time each of the scans took to perform; this needs to be clearly stated when cost benefits are being calculated.

M J WHITTLE

Department of Fetal Medicine, Birmingham Maternity Hospital, Birmingham B15 2TG

1 Luck CA. Value of routine ultrasound scanning at 19 weeks: four year study of 8849 deliveries. BMF 1992;304:1474-8. (6 June.)

EDIToR,-Carole A Luck cannot claim 100\% sensitivity for detecting abnormalities of the renal tract ${ }^{\prime}$ : major congenital abnormalities of the renal tract may be impossible to detect clinically at birth and may not come to light for several months or years. Livera et al scanned 6292 pregnant women at 28 weeks' gestation, looking specifically for abnormalities of the renal tract. ${ }^{2}$ During 18 months' follow up they identified seven babies with abnormalities that had not been detected antenatally. Chitty et al have reported similar findings. ${ }^{3}$ In our unpublished survey of over 10000 deliveries at Torbay Hospital between 1985 and 1989 we discovered six important renal abnormalities during the first year of life in babies who had been clinically normal at birth. All had had routine antenatal ultrasound scanning, four after 19 weeks' gestation.

I would be interested to see an analysis of the follow up of the 88 fetuses with "unilateral hydronephrosis; pelvic diameter 6-10 mm." I suspect that in many this was transient or clinically unimportant pyelectasis.

JOHN BROOMHALL

Torbay Hospital,

Torquay TQ2 7AA

1 Luck CA. Value of routine ultrasound scanning at 19 weeks: four year study of 8849 deliveries. $B M 7$ 1992;304:1474-8. (6 June.)

2 Livera LN, Brookfield DSK, Eggington JA, Hawnaur JM. Antenatal ultrasonography to detect fetal renal abnormalities: prospective screening programme. $B M 7$ 1989;298:1421-3.

3 Chitty LS, Hunt GH, Moore J, Lobb MO. Effectivenes of routine ultrasonography in detecting fetal structura abnormalities in a low risk population. BMF 1991;303:1165-9.

EDIToR,-Carole A Luck fails to discuss what malformations were considered to be detectable by ultrasound scanning before the study started and how the malformations were diagnosed - that is, what the gold standard was for establishing which babies had malformations. ${ }^{1}$ We have to assume that she was trying to diagnose all malformations. If this is the case the overall rate of malformation is low $(166 / 8849)$ and their distribution unusual (for example, 105 of the 166 malformations were malformations of the renal system).

The reason for this is that in most cases the tes being evaluated (ultrasound scanning) was the means by which the malformation was diagnosed (the gold standard) so it is not surprising that the test seemed to perform well. For example, three babies were diagnosed by ultrasound scanning as having posterior urethral valves. At birth none of them had valves, although one of them had appreciable reflux. All three babies are listed in the results as having posterior urethral valves. Eighty eight babies were diagnosed as having mild unilateral hydronephrosis. The proportion of these babies who would have presented in the neonata period with clinical evidence of urinary tract problems cannot be estimated from this study. They are all listed as babies with malformations correctly diagnosed by ultrasound scanning Consequently, for malformations of the rena system ultrasound scanning is reported to have a sensitivity and specificity of $100 \%$ and positive and negative predictive values of $100 \%$ too.

To evaluate properly the performance of antenatal ultrasound scanning in diagnosing malformations there needs to be agreement about which malformations will be assessed as detectable by the test; in addition, the diagnoses must be made with a gold standard that is independent of the scanning -for example, necropsy reports for lethal malformations and independent clinical assessments for non-lethal malformations. If the results of scanning are used to define outcome there is great potential for creating false positive findings. The impact that this has on the babies and their parents must be considered.

Evaluating the effectiveness of routine ultrasound scanning in diagnosing malformations is important. We believe it merits much closer attention to strict methodology.

ROBIN BELL JUDITH LUMLEY

Centre for the Study of Mothers' and Children's Health,

Carlton,

Victoria 3053

Australia

1 Luck CA. Value of routine ultrasound scanning at 19 weeks: four year study of 8849 deliveries. BMF 1992;305:1474-8. (6 June.)

AUTHOR's REPLY,-Our study was prospective, and we were unaware of all the malformations that could be diagnosed when it was initiated. All the lethal anomalies were confirmed at postmortem examination. The non-lethal anomalies were confirmed clinically and by investigation postnatally.

I agree that the sensitivity is distorted by the many minor renal anomalies included in the series and that including sensitivity figures seems to give a bias in favour of a high overall rate of detection of anomalies. For this reason I did not include sensitivities when I initially submitted the paper, but the referee thought they were necessary for statistical reasons.

No child in this four year study has presented to the paediatricians at Heatherwood Hospital after delivery with renal anomalies detectable by ultrasound scanning. The renal disease that has presented has been associated with vesicoureteric reflux and non-dilated systems. I have reviewed the cases of unilateral hydronephrosis detected in 1990. Thirty five cases were found at 19 weeks In two the renal pelvic diameter was $>10 \mathrm{~mm}$ (because of obstruction of the pelviureteric junction in one and non-refluxing, non-obstructive megaureter in the other). In 33 cases the renal pelvic diameter was $<10 \mathrm{~mm}$. It is our policy to scan these infants 72 hours after delivery. If this scan also shows an abnormality investigations are instigated. If the scan is normal the infant has a final scan a 6 weeks. Of the 33 children with pelvic diameter of $6-10 \mathrm{~mm}$, five had obstruction of the pelviureteric junction; three had vesicoureteric reflux; one had a non-refluxing, non-obstructive megaureter; and one had a ureterocele with a dysplastic kidney. suggest to $M \mathrm{~J}$ Whittle that 12 of these children had rather more than minor renal anomalies and that the antenatal ultrasound scans enabled prevention of later morbidity.

The five children with obstruction of the pelviureteric junction $(6-10 \mathrm{~mm})$ at 19 weeks were given prophylactic antibiotics. In one this treatment was stopped after three months and the child presented with a urinary tract infection at 6 months. One child had a breakthrough infection, and another also presented with a urinary tract infection but poor compliance was found.

In the three children thought to have posterio urethral valves antenatally the paediatric urologist considered that there was sufficient diagnostic evidence postnatally to confirm that an appreciable obstruction of the urethra had been present antenatally; "blown valves" were thus diagnosed independently.

I did not wish to mask the fact that only just over a third of cardiac malformations were detected. I tried to emphasise that our scanning policy changed because of the results of the study. Most of the undetected cardiac defects-arose in the firs three years of the four year series, when only four chamber views were examined. Inflow and outflow tracts are now checked as well and, as a result, most of the cardiac defects were detected in the last year of the study.

I apologise for omitting the time taken for each scan and agree that it is important in assessing both performance and cost benefit. We use high quality ultrasound equipment, and 15 minutes is now allowed for each scan at 19 weeks; the actua scanning time is on average 10 minutes per patient.

C A LUCK

Department of Diagnostic Imaging,

Heatherwood Hospital,

Ascot,

Berkshire SL5 8AA

\section{Adjuvant therapy for rectal cancer}

EDIToR,-R H J Begent proposes recommendations for the adjuvant treatment of rectal cancer. These suggest that adjuvant radiotherapy and chemotherapy should be prescribed for tumours of Dukes's stages B and C. The author states that several trials of different aspects of adjuvan chemotherapy and radiotherapy are in progress and that the most constructive approach would be to enter patients into these. I agree with this but believe that there is still insufficient evidence to support the use of adjuvant radiotherapy or radiotherapy and chemotherapy.

There has been a trend towards improved local control in the many published large randomised trials of preoperative or postoperative radiotherapy. Only the European Organisation for Research and Treatment of Cancer study ${ }^{2}$ and the Stockholm study ${ }^{3}$ found a significant difference. Both used preoperative radiotherapy, and included the para-aortic nodes in addition to the pelvis, versus no radiotherapy. In the Stockholm trial there was a significant increase in the incidence of perineal wound infection and dehiscence $(26 \% v$ $19 \%$ ). The postoperative mortality was also significantly higher in the group given preoperative radiotherapy $(8 \% v 2 \%)$, but there was no difference in overall survival. To try to limit morbidity the European Organisation for Research and Treatment of Cancer trial was open only to patients below the age of 70 .

The North Central Cancer Treatment Group's trial is quoted as evidence for a survival advantag associated with combined chemotherapy and postoperative radiotherapy, ${ }^{4}$ and the updated Gastrointestinal Tumour Study Group's study supports this ${ }^{5}$ But the North Central Cancer Treatment Group's study compared radiotherapy alone with the combination of radiotherapy and chemotherapy without a no treatment arm. The Gastrointestinal Tumour Study Group's study did include a no treatment arm as well as chemotherapy alone and radiotherapy alone, and the difference in survival in the group given the combination versus the group given no treatment was significant at seven years. The chemotherapy regimen was toxic and protracted, lasting 18 months. It was poorly tolerated, with only $65 \%$ of patients completing the course and an overall incidence of severe toxicity of $62 \%$.

There are certainly grounds for further study. Though I understand the view that all patients 
with rectal carcinoma of Dukes's stages B and C should receive adjuvant radiotherapy or radiotherapy and chemotherapy, convincing evidence from published randomised trials to support this view is lacking.

Western General Hospital, Edinburgh EH12 5BY

PETER BLISS

1 Begent RHJ. Colorectal cancer. BMf 1992;305:246-9. (25 July ) Gérard A, Buyse M, Norlinger B, Loygue J, Pene F, Kempf $P$, et al. Preoperative radiotherapy as adjuvant treatment in rectal cancer. Ann Surg 1988;208:606-14.

3 Stockholm Rectal Cancer Study Group. Pre-operative short term radiation therapy in operable rectal carcinoma. Cancer 1990; 66:49-55.

4 Krook JE, Moertel CG, Gunderson LL, Wieand HS, Collins RT, Beart RW, et al. Effective surgical adjuvant therapy for highrisk rectal carcinoma. N Engl f Med 1991;324:709-15.

5 Douglass HO, Moertel CG, Mayer RJ, Thomas PRM, Lindblad AS, Mittleman A et al Survival after post operative combinacion ireatment of rectal cancer. NEnglf Med 1986:315:1294-5.

EDrTor,-If local recurrence inevitably afflicted about a third of patients after apparently complete resection of rectal carcinoma, as $\mathrm{R} \mathrm{H}$ J Begent has every reason to write, given some published reports, then the adjuvant radiotherapy and chemotherapy that he recommends for Dukes's stage $B$ and $C$ tumours would make sense.' This outcome, however, may well be avoidable.

I recently examined the sequel in each of the 212 patients with rectal carcinoma under my care during 1985-91 and found no evidence of such depressing failure. Altogether 135 patients had had curative major resections; 41 had palliative removal; and 12 had borderline palliative excision, in which an adjacent structure (usually the uterus or part of the bladder wall) was removed en bloc with the specimen. Of the remainder, 18 had transanal excision and six no procedure. Only one patient was lost to follow up.

None of the patients who had curative resection had adjuvant treatment and local recurrence developed in only three cases; this could be attributed to failure of technique in two cases and to erroneous management in one. None of the patients who had "en bloc" resections received adjuvant treatment either; one developed recurrence-unsurprisingly, since tumour invasion of both the uterus and excised vaginal wall was confirmed on histological examination. Most of the patients who had palliative resections received postoperative radiotherapy; eight developed local recurrence. Twenty two of these 41 patients, however, died within six months of surgery. Similarly, one of 91 patients who had curative resections of tumour of the rectosigmoid during the same period, and one of 11 who had palliative resections, developed local recurrence, though again half died within six months.

Clearly, much hinges on definition and comparing like with like when excluding palliative resections and recognising local recurrence. I define palliative resection of tumour as either cutting or fracturing the tumour off an adjacent irremovable structure, or leaving peritoneal deposits seeded transcoelomically, and local recurrence as the reappearance of tumour at the original site. With these definitions, and if the principles of resection of colorectal cancer are followed-namely, total mesorectal excision for rectal tumours, elimination of exfoliated cancer cells from the bowel lumen above the upper clamps and below the lower clamps before transection, and abdominoperineal excision of rectum when the tumour is too low or poorly differentiated for restorative resectionthen my experience suggests that local recurrence will be so rare that adjuvant treatment is unnecessary.

W H F THOMSON

Gloucester Acute Unit, Gloucestershire Royal Hospital,

Gloucester GLI 3NN

1 Begent RHJ. Colorectal cancer. BMF 1992;305:246-9. (25 July.)

\section{Americans retreat on SI units}

EDIToR,-Magne Nylenna and Richard Smith criticise American doctors' retreat from using Système International (SI) units but do not strengthen their argument by comparing the introduction of a system of units with the global fight against serious disease. ${ }^{.}$The two problems are dissimilar, and it is unfair to make the comparison. Nylenna and Smith do not, however, explore why American readers prefer "conventional" units or mention the partial implementation of SI units in Britain.

Acceptance of SI units implies consistent use of both base units and derived units. Pressures should be expressed in pascals, temperatures in kelvin, and time in seconds. In practice we have a hybrid system of conventional units which often reflect the system used for analysis or measurement - for example, millimetres of mercury for blood pressure. Other compromises are made in expressing the amount of complex biological molecules in mass units rather than moles or as arbitrarily agreed units of biological activity.

If SI units are deemed more scientifically informative it is hard to imagine the day to day advantages in hospital wards of recording temperatures in kelvin, pressures in pascals, and time in seconds. Somewhere the importance of objectives has been overlooked. There seems little point in implementing a system that does not provide an advantage to either the provider or the recipient of health care.

There is no evidence that scientific thought is stifled when SI units are not used-indeed, most major advances in the pure sciences occurred without them. Perhaps the New England fournal of Medicine is still showing leadership qualities by provoking a debate on what we really want of our units in medicine.

\section{Lewisham Hospital}

J C TOWNSEND

London SE13 6LH

1 Nylenna M, Smith R. Americans retreat on SI units. BMF 1992;305:268. (1 August.)

EDIToR, - If Magne Nylenna and Richard Smith's editorial is a plea for standardisation of units and nomenclature' then British journals have lessons to learn. Picking an issue of the $B M \mathcal{F}(25$ July 1992) at random shows several anomalies.

(1) Page 203, column 1, paragraph 3: $1 / \mathrm{m}^{2 /}$ day-some use $L$ as the abbreviation for litre to avoid confusion with the numeral 1 . The superscript notation (i ${ }^{2}$ day ${ }^{1}$ ) is better for complex units. Indeed, some say that for Système International (SI) units the solidus should not be used for "per" at all.

(2) Page 203, column 2, paragraph 3: $n$-methylD-aspartate-should begin with $\mathrm{N}$ (for nitrogen) not $n$ (for normal).

(3) Page 215, abstract: $\mathrm{mm} \mathrm{Hg}$-should be pascals in SI.

(4) Page 216, column 1, paragraph 5: $\mathrm{mM}-$ should be mmoll ' in SI.

(5) Page 216, column 1, paragraph 5: $\mathrm{pH}-$ should be nmol 1 ' hydrogen ion in SI.

If Nylenna and Smith's response to any of the above is that "this is better for our readers" then they should reread the editorial in the New England fournal of Medicine that they criticise so strongly and have some sympathy for the Americans' predicament. ${ }^{2}$ We also question Nylenna and Smith's statement that "Britain ... could introduce SI units everywhere after broad consensus was reached." SI units date from 1960, yet in current British legislation blood and breath ethanol concentrations are cited in $\mathrm{mg}$ and $\mu \mathrm{g}$ per $100 \mathrm{ml}$ respectively. Indeed, the interface between clinical laboratory medicine and other disciplines such as pharmacology and toxicology was largely neglected when SI molar units were introduced in clinical biochemistry.

With SI units (in essence an extension of the metre-kilogram-second system) it is the interpretation that causes all the fuss. There are inconsistencies - for example, one of the base units, the kilogram, is a multiple of the gram. Nevertheless, the framework has stood the test of time, although production of different versions of SI units by different bodies is a continuing source of confusion. Standardisation of SI units is one step. Adoption of molar units to the exclusion of mass units is another step. While doses of drugs, for example, are still measured in mass units it is logical to report plasma concentrations in SI mass units (mgl ', etc) rather than in moles.

The $B M \mathcal{F}$ should lead by example not only in advocating SI units but also in ensuring that the associated conventions are applied sensibly and, if found wanting, are changed.

R J FLANAGAN

Pisons Unit,

Guy's and Lewisham NHS Trust,

London SE14 SER

L F PRESCOTT

Clinical Pharmacology Unit,

University Department of Medicine,

Royal Infirmary,

Edinburgh EH3 9YW

1 Nylenna M, Smith R. Americans retreat on SI units. BMF 1992;305:268. (1 August.)

2 Campion EW. A retreat from SI units. $N$ Engl f Med 1992; 327:49.

\section{Serotonin, gastric emptying, and dyspepsia}

EDITOR,-Functional disorders have important economic and social consequences. ${ }^{1}$ Thus A Chua and colleagues' observation of differences between selected dyspeptic subjects and controls in both solid phase gastric emptying and release of prolactin induced by buspirone is of great interest. ${ }^{2}$ The interpretation of work on functional bowel disorders is confounded by arbitrary definition of cases $^{3}$ and the high prevalence of symptoms in controls who do not present to a doctor. ${ }^{4}$ Explicit criteria for selecting cases and entry criteria are needed for both further research and extrapolation of findings to the clinic or general practice surgery. Unfortunately, Chua and colleagues give few data on the duration and severity of symptoms, or the incidence of other complaints. The proportion of dyspeptic subjects excluded by their complex organic screening process should have been given. There is also no indication of exactly how "formal psychiatric illness" was ruled out. Given the high prevalence of psychological dysfunction in such patients ${ }^{+}$these data are indispensable.

The response of prolactin to serotoninergic agonists has been studied in patients with primary psychiatric disorders. Enhanced release has been found in disorders related to anxiety. ${ }^{5}$ This may also be relevant to work on those labelled as having the postviral fatigue syndrome. ${ }^{6}$ Similar neuroendocrine responses are seen in the premenstrual syndrome, ${ }^{7}$ the postviral fatigue syndrome, ${ }^{6}$ and anxiety disorders. Can changes in stomach motility therefore be attributed to the putative hypersensitivity to serotonin? Do all these conditions produce similar complaints or changes in motility? Moreover, the prolactin response in Chua and colleagues' cases overlaps considerably with that in the controls, from which over half seem indistinguishable; the remainder of the cases are dominated by one particularly high responder. This erodes confidence in the idea that the phenomenon explains symptoms.

Gastric emptying may have been delayed by increased sympathetic tone mediated by anxiety. Alternatively, prolonged loss of appetite due to the patients' symptoms may have resulted in decreased 